\title{
Autoregressive Moving Average with Exogenous Excitation Model for Acous- tic Scattering from Underwater Objects
}

\author{
Lubna Farhi ${ }^{1,}$, Farhan Ur Rehman ${ }^{2}$, Agha Yasir $\mathrm{Ali}^{3}$, \\ ${ }^{1}$ Department of Electronic Engineering, Sir Syed University of Engineering and Technology, Karachi \\ ${ }^{2}$ Department of Mechanical Engineering, University of Toronto, Toronto, Canada \\ ${ }^{3}$ Department of Electronic Engineering, Sir Syed University of Engineering and Technology, Karachi \\ ${ }^{*}$ Corresponding author: agyasir@ssuet.edu.pk
}

\section{Abstract}

This study aims to identify and predict objects underwater using the autoregressive moving average with exogenous excitation (ARMX) model in such a way that the outcome of the model is similar to actual measurements. It is used for parameter estimation. This model is validated by comparing results in actual model with ARMX model, autoregressive with an exogenous variables, and Box Jenkins (BJ) model. The results are analyzed in frequency and time domain by using mean square error criterion. Initial results show that ARMX predicts the acoustic scattering response with an accuracy of $96 \%$, while ARX provides an accuracy of $78 \%$, and BJ model poorly estimates the signal with an accuracy of $35 \%$. ARMX also provides higher accuracy of detection by $7-8 \%$ as compared to the existing techniques.

Keywords-Autoregressive Moving Average with Exogenous Excitation (ARMX), Autoregressive with Exogenous Variables (ARX), Box-Jenkins (BJ) Model.

\section{Introduction}

$\mathrm{T}$ HE field of acoustics studies the propagation and interaction of mechanical waves such as sound waves through different media including air or water. Various methods can be used to model and understand the behavior of those waves including stochastic, deterministic and statistical methods based on underlying physical mechanics of sound waves. Chen et. al. [1] proposed a model for reverberation by using timespace discretization to increase the computation time. A reverberation is the linear response of scatters to the emitting signal and defined by a linear method to attain the impulse response function. Similarly, L. Zao et. al. [2] presented an adaptive noise detection technique for non-stationary acoustic noisy signals. This approach was used in empirical mode decomposition and a vector of Hurst exponent coefficients. The authors worked for UWA DSSS signals detection where they used Lab-VIEW. It incorporates the power spectrum, autocorrelation and cepstrum methods by

ISSN: 2523-0379 (Online), ISSN: 1605-8607 (Print)

DOI: 10.52584/QRJ.1802.23

This is an open access article published by Quaid-e-Awam University of Engineering Science $\&$ Technology, Nawabshah, Pakistan under CC BY 4.0 International License. using the acquisition card and acoustic transducer. Lu et. al. [3] suggested that different detection methods can be independently or jointly used by tuning the display and control parameters in real time. A summary of existing work for signal identification of underwater objects is presented in Table 1. However, all of these approaches usually require some form of propagation model that can provide basic information such as propagation delay and loss. On the other hand, physics-based models incorporate the effects of attenuation, noise, multi-path, Doppler Effect due to object motion, surface waves [4] and bubbles [5] to provide a more accurate depiction of acoustic behavior.

This study provides a unique approach to evaluate modal parameters that result from impulse response from underwater objects in the presence of random excitation and random measured noise as a result of acoustic scattering. The approach involves the use of an autoregressive moving average with exogenous excitation model to predict the contours of a structure while taking random noise and unmeasured excitation into account. Some of the benefits of this technique are that it provides a scaled model shape of the structure and can be used with periodic signals including dif- 
ferent excitation signals. To mitigate the unmeasured excitations, these periodic signals can be combined (with time domain) by synchronous averaging.

This technique requires a multistage estimation algorithm that is first used to evaluate the parameters of the ARMX model and subsequently to compute the modular parameters of the structure. ARMX estimation model uses the position of estimated poles on the Z-plane to find the most suitable model and to discriminate between structural modes and false numerical poles.

This paper is organized as follows. Section 2 mathematically describes the types of models and excitation signals used in the identification process. It also explains the model as a regressive estimator and least square error minimize. Section 3 discusses the simulation results. Section 4 concludes the paper.

\section{Methodology}

Following sections describe the methodology proposed in this paper.

\subsection{Error Estimation Methods}

Error estimation methods aim to identify the system model and minimizing a cost function that represents the expected difference between the actual and estimated output. This estimated output is computed using a predefined model, whose unknown parameters are the optimization variables and the inputs/outputs are in time domain. Mathematically, cost function can be defined as follows [11],

$$
J(\theta)=\sum_{(t=1)}^{(T+\tau)} \| y_{t}-\hat{y}_{(t+\tau / t)}(\theta)
$$

where $\hat{y}_{(t+\tau / t)}(\theta)$ defines the estimated value of the output $y$ at time $t+\tau$, determined using the information available at time $t$ for a model with parameter $\theta$. The fundamental model used for error estimation that the system outputs is found between two input signal $u_{t}$ and the effect of unmeasured noise or disturbances $e_{t}$. Mathematically, Finite Impulse Response (FIR) model is used which is given as follows,

$$
\begin{aligned}
y_{t} & =b_{0} u_{t}+b_{1} u_{(t-1)}++b_{m b} u_{t-m b}+e_{t} \\
& =B\left(q^{-1}\right) u_{t}+e_{t}
\end{aligned}
$$

where $u_{t}=$ input vector and $B\left(q^{-1}\right)=$ FIR kernel:

$$
B\left(q^{-1}\right)=b_{0}+b_{1} q^{-1}+b_{m b} q^{-m b}
$$

Characteristics of models depend on the structure of $B\left(q^{-1}\right)$ and adaptive noise $e_{t}$.

\subsection{ARX Model (Autoregressive with Exogenous Variables)}

The global system characteristics and the coefficients of FIR model are found using the ARX model. ARX model with exogenous input is stated as [4]

$$
A\left(q^{-1}\right) y_{t}=B\left(q^{-1}\right) u_{t}+e_{t}
$$

where,

$$
A\left(q^{-1}\right)=1+a_{1} q^{-1}++a_{m a} q^{-m a}
$$

and $B\left(q^{-1}\right)$ is specified in Equation 2. Therefore, the outcome of the model is given as follows.

$$
y_{t}=\frac{B\left(q^{-1}\right)}{A\left(q^{-1}\right)} u_{t}+\frac{1}{A\left(q^{-1}\right)} e_{t}
$$

The noise is modelled by a factor of $1 / A$ multiplied by the dynamics model. It is important to note that ARX does not model noise and dynamics independently [12].

\subsection{ARMX Model Structure and Estimation Al- gorithm}

ARMX model with variable inputs and noise disturbance signals [13] is given as follows,

$$
\begin{aligned}
& A\left(q^{-1}\right) y_{t}=B\left(q^{-1}\right) u_{t}+C\left(q^{-1}\right) e_{t}, \\
& C\left(q^{-1}\right)=1+c_{1} q^{-1}++c_{m c} q^{-m c},
\end{aligned}
$$

where $A\left(q^{-1}\right)$ is as in Equation 4 and $B\left(q^{-1}\right)$ is given as in Equation 2. The output of this model is given as follows.

$$
y_{t}=\frac{B\left(q^{-1}\right)}{A\left(q^{-1}\right)} u_{t}+\frac{C\left(q^{-1}\right)}{A\left(q^{-1}\right)} e_{t}
$$

Equation error methodology in ARMX algorithm detects the output, which is a summation of three regression terms: past inputs, outputs and white noise. The inputs of model are the combination of observed input $\left(u_{t}\right)$ which gives the deterministic component by transfer function $\frac{B\left(q^{-1}\right)}{A\left(q^{-1}\right)}$; however, its output $y_{0}(t)$ is not available. The other input, which is known as white noise $\left(e_{t}\right)$, helps to determine the stochastic component by $\frac{C\left(q^{-1}\right)}{A\left(q^{-1}\right)}$ transfer function and its output is a noise $\nu(t)$ that signifies the effect of white noise [14]. The observed output is given as follows.

$$
\tilde{y}_{0}(t)=y_{0}(t)+\nu(t)
$$

In ARMX, the dynamics of the noise that are parameterized are more flexible than in the ARX model. ARMX extends the ARX structure by providing more flexibility for modeling noise using the $\mathrm{C}$ parameters (a moving average of white noise). This allows ARMX to be the preferable option when the input is dominated by the disturbances known as load disturbances 


\begin{tabular}{|l|l|l|}
\hline Author (Year) & Paper Title & Methodology \\
\hline Dmitrieva M., et. al. (2019) [6] & $\begin{array}{l}\text { "Material recognition based on the } \\
\text { time delay of secondary reflections } \\
\text { using wideband sonar pulses". }\end{array}$ & $\begin{array}{l}\text { Methodology using wideband chirp pulses. } \\
\text { The accuracy of identification was } 83.5 \% \\
\text { for the shell material and 79.9\% for filler } \\
\text { material on the synthetic data. }\end{array}$ \\
\hline Anitha, U., et. al. (2018) [7] & $\begin{array}{l}\text { "Underwater object identification and } \\
\text { recognition with sonar images using } \\
\text { soft computing techniques". }\end{array}$ & $\begin{array}{l}\text { Approach based on "Adaptive-Neuro Fuzzy } \\
\text { Inference System"for underwater object } \\
\text { detection by using sonar and claims for better } \\
\text { results. }\end{array}$ \\
\hline Jia H., et. al. (2017) [8] & $\begin{array}{l}\text { "Rigid and elastic acoustic scattering } \\
\text { signal separation for underwater target" }\end{array}$ & $\begin{array}{l}\text { Fractional Fourier transform (FRFT) is used } \\
\text { to separate the target rigid and elastic } \\
\text { acoustic scattering components. }\end{array}$ \\
\hline Josso NF., et. al. (2011) [9] & $\begin{array}{l}\text { "Nonstationary System Analysis Methods } \\
\text { for Underwater Acoustic Communications" }\end{array}$ & $\begin{array}{l}\text { Presented two approaches. First methodology } \\
\text { is "canonical time-scale channel model and } \\
\text { "ray system model" }\end{array}$ \\
\hline Li W., et. al. (2004) [10] & $\begin{array}{l}\text { "Size identification of underwater objects } \\
\text { from backscattering signals of arbitrary } \\
\text { looking angles." }\end{array}$ & $\begin{array}{l}\text { The approach uses various frequency ranges to } \\
\text { find different parameters Authors claims } \\
\text { that proposed method achieved better accuracy } \\
\text { then other state of art methods }\end{array}$ \\
\hline
\end{tabular}

TABLE 1: Summary of the existing work

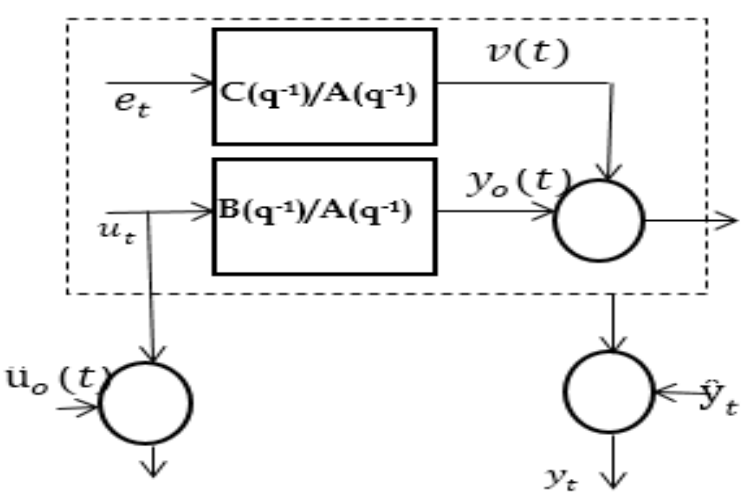

Fig. 1: ARMX model

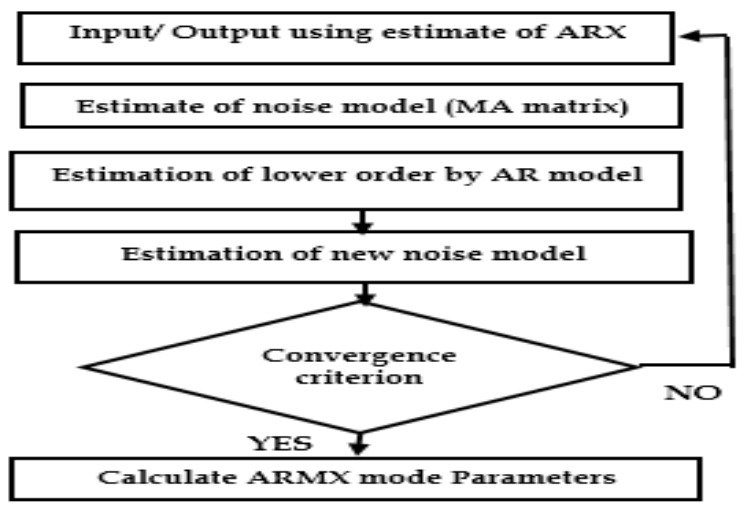

Fig. 2: Block diagram of ARMX estimation algorithm

[15][20]. One of the major advantages of autoregressive (AR) based representation is that the resonant frequencies can be accurately localized and detected. In order to obtain the elastic and geometrical properties, the resonant frequencies are clustered together in the form of identifiable wave families that are then classified with respect to scattered waves using resonance scattering theory [16].

\section{Box-Jenkins (BJ) Model}

Polynomial model and it is written as:

$$
y_{t}=\frac{B\left(q^{-1}\right)}{A\left(q^{-1}\right)} u_{t}+\frac{C\left(q^{-1}\right)}{A\left(q^{-1}\right)} e_{t}
$$

where,

$$
\left\{\begin{array}{l}
D\left(q^{-1}\right)=1+d_{1} q^{-1}++d_{m d} q^{-m d} \\
F\left(q^{-1}\right)=1+f_{1} q^{-1}++f_{m f} q^{-m f}
\end{array}\right.
$$

Equation 11-12 characterize the dynamics and noise using rational polynomial functions. As a result, the BJ model is a better option when the input is not dominated by noise. Instead, the noise is a primary measurement disturbance that is added afterwards. This structure allows more flexibility to model noise eue to its ability to independently model noise. The input-output relationship can take the following form,

$$
y(K)=\psi(k)^{T} \theta
$$

where, $\psi(k)$ is the regression vector of $y$ and $u$ and a function of past data. $\theta \in D M \subset R P$ is a parameter vector to estimate the model [17].

\section{Simulation \& Results}

The parameters of the ARMX model can now be chosen through a multistage estimation algorithm that chooses the best-fit model from the estimated models based on the position of their corresponding estimated 


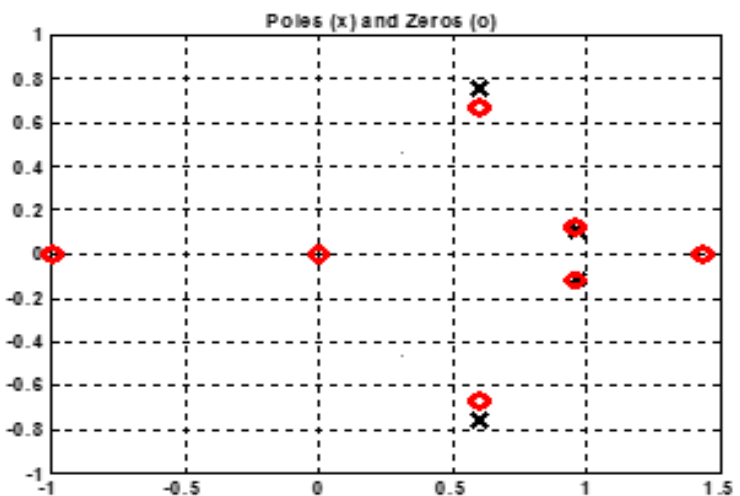

Fig. 3: Estimated Poles and Zeros on Z-Plane

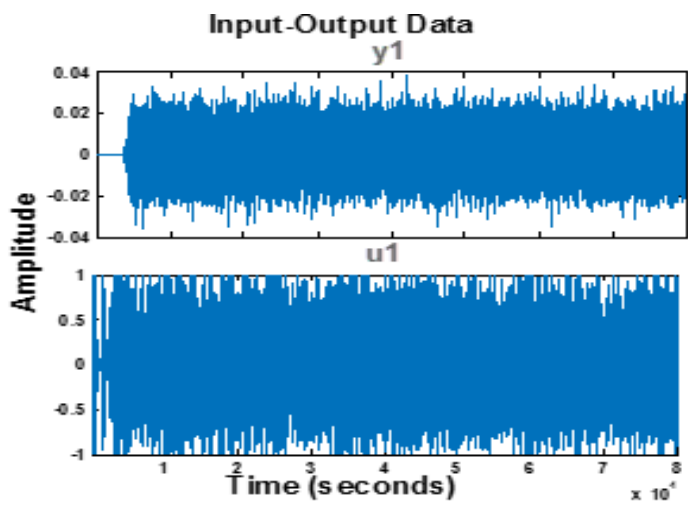

Fig. 4: Acoustic signals: Input and Output

poles on the Z-plane as shown in Fig ure 3. Once the ARMX parameters have been chosen, the model parameters of the structure can finally be calculated. The complicated or noisy signals are represented in Figure 4. It is difficult to optimize the polynomial orders in ARMX model. It is the most suitable and immediate method for finding the poles of a system and scattering the resonance frequencies. In the proposed methodology, ARMX technique includes zeros in the parametric model, allowing for a more significant and accurate data representation.

In order to optimize the computation of the model, an assessment of time and frequency correlation properties of the path coefficients are used. The ARMX,

\begin{tabular}{|l|l|l|}
\hline \multirow{3}{*}{$\begin{array}{l}\text { Input } \\
\text { Parameters }\end{array}$} & Sample time & 1 seconds \\
\cline { 2 - 3 } & Polynomial orders & $\begin{array}{l}N_{a}=2, N_{b}=2 \\
N_{c}=2, N_{k}=2\end{array}$ \\
\cline { 2 - 3 } & Free coefficients & 06 \\
\cline { 2 - 3 } $\begin{array}{l}\text { Output } \\
\text { Parameters }\end{array}$ & $\begin{array}{l}\text { Fit to estimation } \\
\text { data(Accuracy) }\end{array}$ & 14 \\
\cline { 2 - 3 } & $\begin{array}{l}\text { Mean square } \\
\text { error (MSE) }\end{array}$ & $96.42 \%$ \\
\hline
\end{tabular}

TABLE 2: ARMX model generated parameters

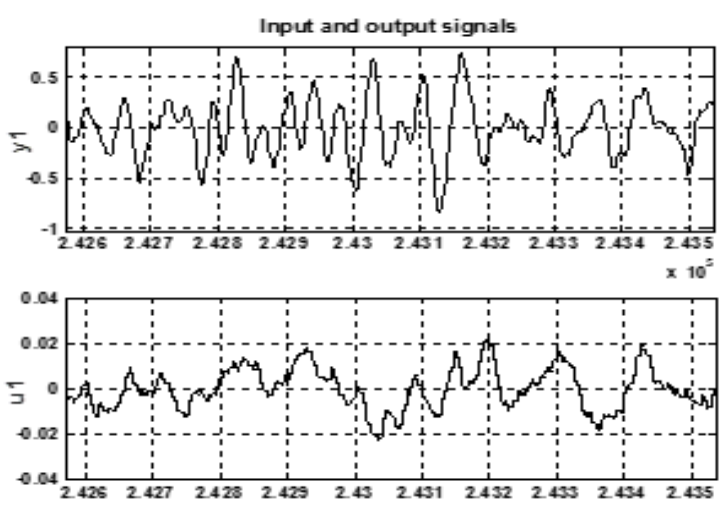

Fig. 5: Input and Output de-trended data, data has zero mean value

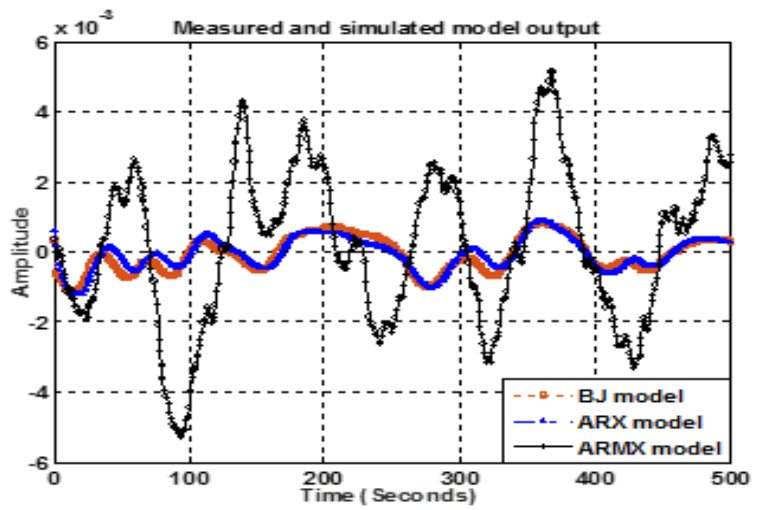

Fig. 6: Measured and Simulated output of Models

ARX and BJ models are finally validated using real life data from four independent experiments. Specifically, experimental data is used to assess the statically and the auto-correlation functions of the large-scale loss and the short-term path gains. ARMX extends the ARX structure by providing more flexibility for modeling the trended noise as shown in Figure 5, using the $\mathrm{C}$ parameters (a moving average of white noise). This allows ARMX to be the preferable option when the input is dominated by disturbances. Figure 6 shows the results of ARMX, ARX AND BJ models outputs. Varying the parameters and comparing the performance of all three models, i.e., ARX, BJ and ARMX, simulation results in Table 3 show that ARMX is the best-fit model and more accurate as compared to other parametric models. The ARMX

\begin{tabular}{|l|l|}
\hline Model Type & Output Accuracy (\%) \\
\hline ARMX (Proposed) & 97.8 \\
\hline ARX & 78.5 \\
\hline BJ & 35.37 \\
\hline
\end{tabular}

TABLE 3: Accuracy comparison 


\begin{tabular}{|l|l|l|}
\hline No. & Model Type & $\begin{array}{l}\text { Output } \\
\text { Accuracy (\%) }\end{array}$ \\
\hline 1. & $\begin{array}{l}\text { ARMX model } \\
\text { (Proposed) }\end{array}$ & 97.8 \\
\hline 2. & $\begin{array}{l}\text { Wideband sonar } \\
\text { pulses method [6]. }\end{array}$ & 78.5 \\
\hline 3. & $\begin{array}{l}\text { Canonical time-scale } \\
\text { channel model [9]. }\end{array}$ & 86.7 \\
\hline 4. & $\begin{array}{l}\text { Sonar images using } \\
\text { soft computing } \\
\text { techniques [7]. }\end{array}$ & 89.7 \\
\hline
\end{tabular}

TABLE 4: Accuracy comparison with the existing techniques

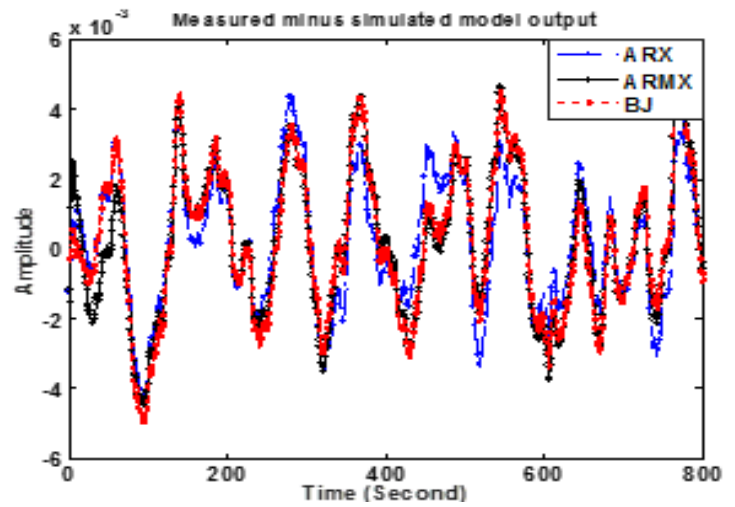

Fig. 7: Error between measured and simulated model output.

algorithm was developed to yield more accurate modal parameters than ARX models estimated using the least squares criterion that included unmeasured excitation, Error estimation is shown in Figure 7 by least square method.

As shown in Figure 8, amplitude bode plot, the resonant frequency peaks for the ARMX model can be easily identified as sharp distinct features; whereas, in ARX and BJ models, the plot is smoother and the resonant frequency is difficult to distinguish.

The spectrum is only plotted for frequencies smaller than the Nyquist frequency. Table 4 shows that the results improve by using ARMX model as compared to the existing techniques.

\section{Conclusion}

After comparing the results of simulations including frequency diagrams, parameter estimations, pole-zero analyses and auto and cross correlations, it can be concluded that the best-fit model was provided by ARMX. This model can be described by 6 parameters with a delay of one sample. As a result, the ARMX model provides an accuracy of $97 \%$ which is much higher than the corresponding accuracy of the ARX model (78\%)

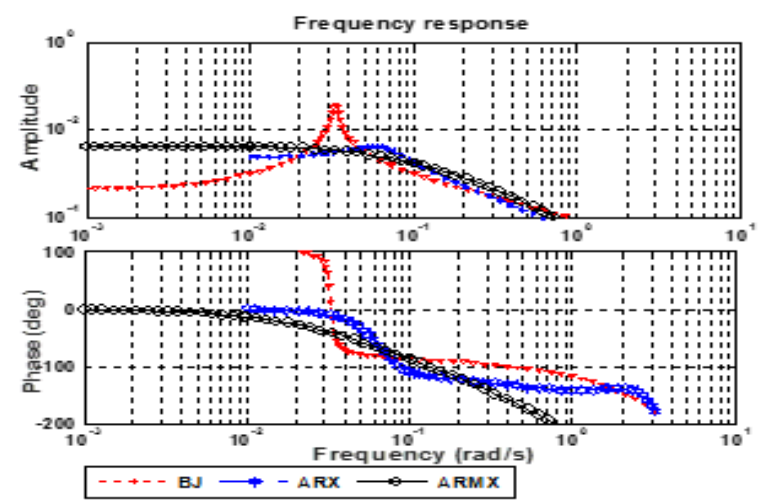

Fig. 8: Frequency Response of ARMX, ARX and BJ models

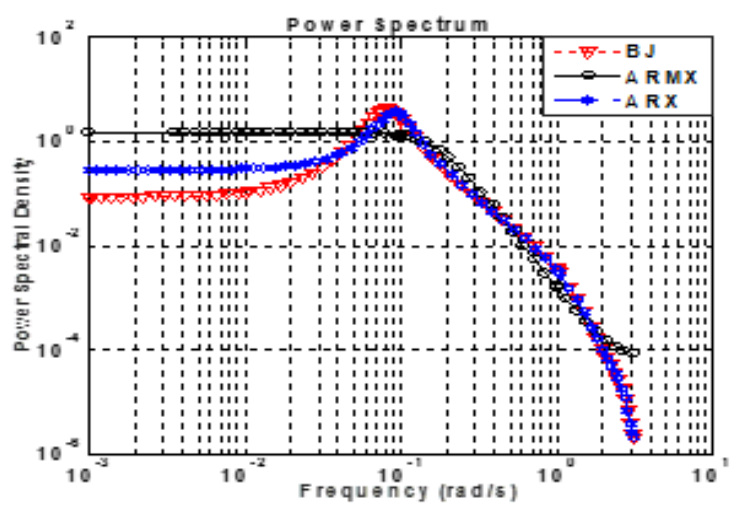

Fig. 9: Power spectrum (db) of frequency signal

and the BJ model (35\%). Therefore, the accuracy from ARMX model was improved by $19.3 \%$ as compared to ARX model, and by $62 \%$ as compared to BJ model. In addition, the detection accuracy improved by 7 $8 \%$ by using ARMX model in coparison with the exising techniques. A 97\% accuracy of estimation can be deemed as a successful identification of the real object based on acoustics. The results showed that ARMX is best linear model because of its low number of parameters and higher percentage of accuracy.

This model will be validated in future by conducting a probe analysis experiment. It is intended to consolidate the idea that ARMX can realistically provide a higher accuracy as compared to the state-of-the-art techniques used for underwater object detection

\section{References}

[1] Wenjian C, Hui S, Minghui Z, Guangping Z, Gen L.," A model for numeri-cal simulation sea bottom reverberation using time-space discretization", In-ternational Conference on Computer Application and System Modeling, IEEE, Vol. 10, 210-240, 2010. 
[2] L. Zão and R. Coelho, "Empirical signal decomposition for acoustic noise detection," IEEE Sensor Array and Multichannel Signal Processing Work-shop (SAM), Rio de Janerio, pp. 1-5, 2010.

[3] L. Ma, C. Fan, W. Sun and G. Qiao, "A Real-Time Detection System for Non-Cooperative Underwater Acoustic DSSS Signals Based on LabVIEW", 2nd IEEE Advanced Information Management,Communicates,Electronic and Automation Control Conference (IMCEC), pp. 1331$1335,2018$.

[4] E. Demirors, G. Sklivanitis, T. Melodia, S. N. Batalama and D. A. Pados, "Software-defined underwater acoustic networks: toward a high-rate real-time reconfigurable modem," in IEEE Communications Magazine, vol. 53, pp. 64$71,2015$.

[5] L. Krishnan, T. Betlehem and P. D. Teal, "A Statistically Robust Approach to Acoustic Impulse Response Shaping" , IEEE Signal Processing Letters, vol. 24, pp. 1138-1142, 2017.

[6] ] Dmitrieva M, Brown K, Heald G, Lane D., "Material recognition based on the time delay of secondary reflections using wideband sonar pulses", IET Radar, Sonar \& Navigation, vol. 13, pp. 2034-40, 2019.

[7] Anitha, U., and S. Malarkkan., "Underwater object identification and recog-nition with sonar images using soft computing techniques", Indian journal of geo-marine sciences, pp. 665-673, 2018.

[8] Beran, J., "Maximum likelihood estimation of the differencing parameter for invertible short and long memory autoregressive integrated moving average models", Journal of the Royal Statistical Society: Series B (Methodological), vol. 57, pp. 659-672, 1995.

[9] Z.M.Yusoff, Z.Muhammad, M.H.F. Rahiman, M.N. Taib, "ARX Mod-eling for Down-Flowing Steam Distillation System", IEEE 8th Interna-tional Colloquium on Signal Processing and its Application, Melaka, Malaysia, 2012.

[10] Ljung, L., "System identification", Wiley Encyclopedia of Electrical and Electronics Engineering, 2017.

[11] Chitre, M., "A high-frequency warm shallow water acoustic communica-tions channel model and measurements", The Journal of the Acoustical So-ciety of America, vol. 122, no. 5, pp. 2580-2586, 2007.

[12] Siderius, M. and Porter, M.B.," Modeling broadband ocean acoustic trans-missions with time-varying sea surfaces", The Journal of the Acoustical So-ciety of America, vol. 124, no. 1, pp. 137-150, 2008.

[13] Karjadi, E.A., Badiey, M. and Kirby Jr, J.T.,"Impact of surface gravity waves on high-frequency acoustic propagation in shallow water", The Journal of the Acoustical Society of America, vol. 127, no. 3, pp. 1787-1787, 2010.

[14] Moore, S.M., Lai, J.C.S. and Shankar. K, "ARMAX modal parameter identi-fication in the presence of unmeasured excitation-I: Theoretical back-ground". Mechanical systems and signal processing, vol. 21, no. 4, pp. 1601-1615, 2006.

[15] Ljung, L., "System identification", Wiley Encyclopedia of Electrical and Electronics Engineering, 2017.

[16] Mohanty, P. and Rixen, D.J., "Operational modal analysis in the presence of harmonic excitation", Journal of sound and vibration, vol. 270, pp. 93-109, 2004.

[17] Pintelon, R., Peeters, B. and Guillaume, P.," Continuoustime operational modal analysis in the presence of harmonic disturbances", Mechanical Sys-tems and Signal Processing, vol. 22, no. 5, pp. 1017-1035, 2008.

[18] Jia H, Li X, Meng X., "Rigid and elastic acoustic scattering signal separation for underwater target", The Journal of the
Acoustical Society of America, vol. 142, no. 2, pp. 653-65., 2017.

[19] Josso NF, Zhang JJ, Papandreou-Suppappola A, Ioana C, Duman TM, "Nonstationary system analysis methods for underwater acoustic com-munications", EURASIP Journal on Advances in Signal Processing, pp. 1-4, 2011.

[20] Li W, Liu GR, Zhang XM. "Size identification of underwater objects from backscattering signals of arbitrary looking angles". Journal of Computation-al Acoustics, vol. 2, no. 3, pp. 1-17, 2004. 